\title{
Topology optimization of a pseudo 3D thermofluid heat sink model
}

\author{
Haertel, Jan H. K.; Engelbrecht, Kurt; Lazarov, Boyan S.; Sigmund, Ole
}

Published in:

International Journal of Heat and Mass Transfer

Link to article, DOI:

10.1016/j.ijheatmasstransfer.2018.01.078

Publication date:

2018

Document Version

Peer reviewed version

Link back to DTU Orbit

Citation (APA):

Haertel, J. H. K., Engelbrecht, K., Lazarov, B. S., \& Sigmund, O. (2018). Topology optimization of a pseudo 3D thermofluid heat sink model. International Journal of Heat and Mass Transfer, 121, 1073-1088.

https://doi.org/10.1016/j.ijheatmasstransfer.2018.01.078

\section{General rights}

Copyright and moral rights for the publications made accessible in the public portal are retained by the authors and/or other copyright owners and it is a condition of accessing publications that users recognise and abide by the legal requirements associated with these rights.

- Users may download and print one copy of any publication from the public portal for the purpose of private study or research.

- You may not further distribute the material or use it for any profit-making activity or commercial gain

- You may freely distribute the URL identifying the publication in the public portal 


\title{
Topology Optimization of a Pseudo 3D Thermofluid Heat Sink Model
}

\author{
Jan H. K. Haertel ${ }^{1 \mathrm{a}}$, Kurt Engelbrecht ${ }^{\mathrm{a}}$, Boyan S. Lazarov ${ }^{\mathrm{b}}$, Ole Sigmund ${ }^{\mathrm{b}}$ \\ ${ }^{a}$ Department of Energy Conversion and Storage, Technical University of Denmark, Frederiksborgvej 399, 4000 \\ Roskilde, Denmark \\ ${ }^{b}$ Department of Mechanical Engineering, Technical University of Denmark, Nils Koppels Allé Building 404, 2800 \\ Kgs. Lyngby, Denmark
}

\begin{abstract}
This paper investigates the application of density-based topology optimization to the design of air-cooled forced convection heat sinks. To reduce the computational burden that is associated with a full 3D optimization, a pseudo 3D optimization model comprising a 2D modeled conducting metal base layer and a thermally coupled 2D modeled thermofluid design layer is used. Symmetry conditions perpendicular to the flow direction are applied to generate periodic heat sink designs. The optimization objective is to minimize the heat sink heat transfer resistance for a fixed pressure drop over the heat sink and a fixed heat production rate in the base plate. Optimized designs are presented and the resulting fin geometry is discussed from a thermal engineering point of view and compared to fin shapes resulting from a pressure drop minimization objective. Parametric studies are conducted to analyze the influence of the pressure drop on the heat sink heat transfer resistance. To quantify the influence of the assumptions made in the pseudo 3D optimization model, validation simulations with a body-fitted mesh in 2D and 3D are conducted. A good agreement between optimization model and validation simulations is found, confirming the physical validity of the utilized optimization model. Two topology optimized designs are exemplarily benchmarked against a size optimized parallel fin heat sink and an up to $13.6 \%$ lower thermal resistance is found to be realized by the topology optimization.
\end{abstract}

Keywords: Topology optimization, Heat sink design, Thermofluid modeling, Forced convection

\section{Introduction}

Forced convection heat sinks are used in a wide range of applications. This paper focuses on the design of air-cooled heat sinks as used for micro-electronics cooling since thermal management is increasingly becoming a bottleneck for advancement in the design of these systems [1]. Moreover,

5 better heat management allows electronics to operate at higher performance for longer periods of time 2]. Classical heat sink designs applied to electronics cooling are mainly pin fin and parallel

${ }^{1}$ Corresponding author: jhkh@dtu.dk (Jan H. K. Haertel) 


\begin{tabular}{|c|c|c|c|}
\hline \multicolumn{4}{|c|}{ Nomenclature } \\
\hline$A_{\Omega_{d}}$ & design domain area & \multicolumn{2}{|c|}{ Abbreviations } \\
\hline$b_{j}$ & convexity parameter in interpolation & $2 \mathrm{D}$ & two-dimensional \\
\hline & function & & three-dimensional \\
\hline$c$ & heat capacity & $\min$ & minimize \\
\hline$C_{j}$ & parameter in RAMP interpolation & PDE & partial differential equation \\
\hline$D a$ & Darcy number & s.t. & subject to \\
\hline $\mathbf{F}$ & volumetric force & & \\
\hline$f$ & fin volume fraction & \multicolumn{2}{|c|}{ Greek symbols } \\
\hline$h$ & heat transfer coefficient & $\bar{\alpha}$ & maximum inverse permeability \\
\hline$I_{\alpha}$ & friction interpolation function & $\beta$ & projection steepness parameter \\
\hline$I_{h}$ & heat transfer interpolation function & $\gamma$ & design variable \\
\hline$I_{k}$ & conductivity interpolation function & $\tilde{\gamma}$ & filtered design variable \\
\hline$k$ & thermal conductivity & $\overline{\tilde{\gamma}}$ & projected design variable \\
\hline$L_{c}$ & characteristic length & $\Gamma_{j}$ & domain boundary $\mathrm{j}$ \\
\hline $\mathrm{n}$ & normal vector & $\eta$ & projection threshold parameter \\
\hline$n_{\text {eval }}$ & number of model evaluations & $\mu_{f}$ & dynamic fluid viscosity \\
\hline$p$ & pressure & $\rho_{f}$ & fluid density \\
\hline$\dot{q}_{\text {inter }}$ & $\begin{array}{l}\text { transferred heat between base } \\
\text { plate and design layer }\end{array}$ & $\Omega_{j}$ & domain $\mathrm{j}$ \\
\hline$\dot{Q}_{\text {prod }}$ & produced heat in base plate & \multicolumn{2}{|c|}{ Subscripts } \\
\hline $\mathbf{r}$ & $\begin{array}{l}\text { residual of discretized multiphysics } \\
\text { problem }\end{array}$ & $\begin{array}{l}\text { air } \\
\text { av }\end{array}$ & $\begin{array}{l}\text { air } \\
\text { average }\end{array}$ \\
\hline$r_{\text {filter }}$ & filter parameter & $d$ & design domain \\
\hline$R_{t h}$ & thermal resistance & $f$ & fluid \\
\hline $\mathrm{s}$ & state vector of multiphysics problem & $i$ & counter subscript \\
\hline$T$ & temperature & in & inlet \\
\hline $\mathbf{u}$ & velocity vector & $j$ & counter subscript \\
\hline$V_{\text {base plate }}$ & volume base plate & $\max$ & maximum \\
\hline $\mathbf{x}$ & xy-coordinate vector & out & outlet \\
\hline$\Delta z_{\text {base plate }}$ & thickness base plate & $s$ & solid \\
\hline$\Delta z_{\text {channel }}$ & thickness channel & $\begin{array}{l}\text { symm } \\
w\end{array}$ & $\begin{array}{l}\text { symmetry } \\
\text { wall }\end{array}$ \\
\hline
\end{tabular}

plate fin designs often with air as the coolant due to availability, simplicity of operation and low cost. Also microchannel heat sinks cooled by liquids such as water and oil have been investigated in various works as they allow for the rejection of higher specific power rates than air-cooled heat sinks. A comprehensive review of microchannel heat sinks considering channel geometry, flow conditions, and utilized coolants is given in [3]. The heat transfer and fluid dynamics in heat sinks are for example described in [4. Various later works deal with the design and optimization of forced convection heat sinks: for instance Lee et al. [5] apply a simulation model based on analytical equations to the prediction and optimization of the thermal performance of fin heat sinks. Park et al. 6] use the results of 3D CFD simulations to create a Kriging metamodel which is used for shape optimization of a plate fin heat sink. With the above mentioned methods significant improvements in the thermal design of heat sinks can be achieved; however, they are limited in the sense that an 
a priori design parametrization is needed both for the fin shape and the position of the fins relative to each other. In contrast, topology optimization allows for almost unlimited design freedom which makes it possible to identify also unintuitive and complex optimized structures without relying on the design engineer's intuition. This aspect becomes even more important with the increasing maturing of additive manufacturing technologies as these methods provide unprecedented design freedom.

Topology optimization [7] was developed in the context of structural engineering but has since then been applied to a wide range of engineering disciplines such as acoustics, photonics, and fluid flow [8]. It is a means to optimize the material distribution in a given design domain subject to certain constraints. In density-based topology optimization, which is used in this work, a density-field is introduced that can take the value of 0 (solid) or 1 (void) in each point of a design domain. This binary optimization problem is relaxed to continuous values between 0 and 1 to allow for the use of efficient gradient-based optimization techniques. In the presented thermofluid design problem 0 corresponds to heat sink material and 1 to fluid passage, thus allowing for a flexible representation of arbitrary heat sink fin geometries during the optimization. Topology optimization applied to the design of thermal systems such as heat sinks and heat exchangers is an active field of research [9. Early applications of topology optimization to heat transfer problems consider 2D heat conduction problems with convective heat transfer to an ambient fluid in the out-of-plane direction by assuming a constant heat transfer coefficient as e.g. [10]. When treating $2 \mathrm{D}$ conduction problems with convective heat transfer within the modeled plane, the design dependent convective boundary to the fluid needs to be captured which can be done by using an interpolation scheme [11, 12, 13, by applying level set based topology optimization to track the boundary [14, or by comparing the density of adjacent elements in the finite element mesh [15]. A constant heat transfer coefficient is assumed in [11, 12, 14] whereas [13] and [15] use a surrogate model for the heat transfer coefficient to capture the dependence of the local convective heat transfer on the geometry of the optimized structure to some degree. More recently published works present also 3D optimization models with diffusive heat transport in the solid and design dependent convective boundaries with a constant heat transfer coefficient using densitybased [16, 17] and level set [18, topology optimization. Dede and coworkers [16] use additive layer manufacturing to fabricate and subsequently experimentally evaluate an optimized heat sink design. The thermal integration of a thermoelectric cooler in a robotic downhole intervention tool using topology optimization to distribute conducting and insulating material in a 3D domain as well as fabrication and experimental validation of the prototype is presented in [19. Pizzolato et al. 20] apply density-based topology optimization to the design of conducting fins in a phase change material (PCM) storage tank modeling the solidification of the PCM as transient thermal diffusion problem both in $2 \mathrm{D}$ and $3 \mathrm{D}$. 
The topology optimization works presented above simplify the heat transfer to the ambient fluid to Newtons law of cooling, i.e. assuming a constant heat transfer coefficient or a surrogate model for it. This limiting assumption can be avoided when using thermofluid, or conjugate heat transfer, topology optimization models which also explicitly consider the heat transfer in the fluid during the optimization. First fairly academic applications of topology optimization to 2D forced convection conjugate heat transfer problems are given in 21, 22. 2D topology optimization of water-cooled microchannel heat sinks is presented in [23] solving the Navier-Stokes equations and 24] assuming Stokes flow where 24 fabricate and experimentally evaluate an optimized heat sink prototype. Matsumori et al. 25] apply topology optimization to a 2D thermofluid heat exchanger model; however, assuming the same thermal conductivity in solid and fluid. Similar heat exchanger models are treated in [26] that apply 2D lattice Boltzmann modeling and [27] who use a level set topology optimization approach to generate optimized designs in 2D and 3D. Qian and Dede [28] present a thermofluid model for topology optimization under tangential thermal gradients. The abovementioned works on thermofluid topology optimization, except for [27] that also conduct 3D optimizations, rely on 2D optimization models. This approach was extended by 29] and later [30] to a pseudo 3D model with a heat sink base plate thermally interacting with 70 a thermofluid design layer that represents the heat sink fins and fluid flow. The extension of the presented work which also deals with a pseudo 3D heat sink model compared to [29, 30] is discussed later in this section. A fully developed flow heat exchanger model is given in 31] where the fluid flow is perpendicular to the design domain. All above presented works on thermofluid topology optimization treat laminar flow problems. Turbulent flow topology optimization is still in its very beginnings and, to the authors' best knowledge, only Kontoleontos et al. 32] have applied turbulent flow topology optimization to a conjugate heat transfer problem, albeit with the simplification of not considering the temperature field in the solid. Natural convection problems have only more recently been investigated due to the complex coupling of fluid and temperature problem. Alexandersen et al. 33] pioneered this area presenting a 2D natural convection heat sink topology optimization model that was later extended to 3D [34 and large-scale 3D [35. Level set based topology optimization applied to $3 \mathrm{D}$ and $2 \mathrm{D}$ transient natural convection problems is treated in [36].

Full 3D optimization models allow to exploit 3D design freedom; however, at the cost of high computational demand. Conducting such optimizations is currently only possible using a computer cluster and parallelizable optimization models [35]. The computational effort is significantly reduced when using a pseudo $3 \mathrm{D}$ heat sink model comprised of the $2 \mathrm{D}$ modeled base plate and 2D thermofluid design layer while it allows for more detailed modeling than pure 2D heat sink topology optimization models. Such pseudo 3D models can be optimized on a powerful desktop computer and, therefore, be applied by a broader range of users than models which require access 
to high performance computing tools. Steady state laminar flow pseudo 3D heat sink models, as analyzed in this study, have been treated before in conference papers [29, 30]; however, without detailed discussion of the results. Furthermore, full 3D validation simulations that can be used to assess assumptions regarding the heat transfer between base plate and thermofluid design layer and other simplifications have not yet been conducted. This is done in the presented study and considered one of the main contributions of this work. Moreover, the validation simulations are conducted using a body-fitted mesh and explicit representation of the solid-fluid boundary to assess the accuracy of the implicit solid-fluid representation based on a density field which inherently has some grey area at the solid-fluid interface. This has previously been done in [25] for forced convection conjugate heat transfer topology optimization; nevertheless, only for one exemplary design in 2D and assuming equal thermal conductivity in the solid and fluid. In this work, 2D and $3 \mathrm{D}$ validation simulations are conducted over the entire range of analyzed pressure drops to assess the difference between the optimization model, 2D body-fitted mesh validation model, and 3D body-fitted mesh validation model separately. A novelty for thermofluid topology optimization in this work is the application of symmetry conditions at the boundaries perpendicular to the flow direction to generate periodic heat sink designs. Furthermore, this is the first forced convection thermofluid topology optimization study to use air as a coolant, which is challenging for the optimization due to the large conductivity difference between air and metal, apart from 31] that rely on a simplified fully developed flow model. Also, analyzing differences in fin shapes between thermal resistance and pressure drop minimization, as done in this study, is a novelty in works on topology optimization. Two exemplary topology optimized designs are compared to size optimized parallel fin designs to provide a quantitative benchmarking against a simpler established heat sink geometry. A comparable benchmarking has so far in studies on thermofluid topology optimization only been conducted for the simplified fully developed flow model treated in [31.

\section{Heat sink model}

\subsection{General model description}

A 3D sketch of a forced convection heat sink as treated in this work can be seen in Fig. 1 (left). Heat is generated in the heat sink base plate and transferred to the fluid flowing between the fins. Since topology optimization typically requires several hundred iterations until convergence to a final design, a full 3D optimization is computationally expensive. Hence, a pseudo 3D heat sink optimization model, as shown in Fig. 1 (right), which approximates the original 3D heat sink model as two 2D thermally coupled problems is used in this work. The simplified model consists of a 2D thermofluid design layer that represents the original 3D thermofluid problem in the fluid flow and heat sink fins and of a $2 \mathrm{D}$ conductive base plate layer that represents the original $3 \mathrm{D}$ thermal 
diffusion problem in the heat sink base plate. The modeling of the heat transfer between the two coupled layers is described in section 2.2.2. Two different thermofluid design layer geometries, corresponding to heat sink model $a$ and model $b$, are shown in Fig. 2 The respective design layer consists of the design domain, $\Omega_{d}$, and non-optimizable pure fluid area, $\Omega_{f}$. As mentioned above, the design variable field can represent either heat sink fin material or fluid passage at each point of the design domain; thus, allowing for a flexible optimization of the fin topology. The heat sink base plate is below the design domain and has the same fixed area as $\Omega_{d}$. The modeling of the thermal coupling between the base plate and thermofluid design layer is described in section 2.2.2 Figure 2 states additionally the boundary conditions and dimensions of model $a$ and model $b$. For both $2 \mathrm{D}$ models and the corresponding $3 \mathrm{D}$ validation models, a channel height, $\Delta z_{\text {channel }}$, of $8 \mathrm{~mm}$ and a thickness of the metal base plate, $\Delta z_{\text {base plate }}$, of $0.2 \mathrm{~mm}$ are assumed. However, in section 5.2.5, a reduced base plate thickness of $0.005 \mathrm{~mm}$ is analyzed in order to emphasize hotspots. Symmetry conditions are applied at the top and bottom boundary of model a which leads to a periodic heat sink design perpendicular to the air flow direction. In model $b$, symmetry is assumed only at the top boundary of the design domain so that the modeled area corresponds to the bottom half of the entire heat sink. This second non-periodic heat sink geometry with inlet and outlet width smaller than the design domain width represents a design problem where the best design is less intuitive, although it does not necessarily represent a practical application. This case is optimized to demonstrate the potential of the topology optimization method to generate more complex designs and to show a case where the optimal number of fins of the design changes for different pressure drops over the heat sink.

\subsection{Thermofluid modeling}

Throughout this work, an incompressible, laminar, and steady-state flow is assumed. Furthermore, as described in the previous section, the fluid and heat transfer problems in the thermofluid design layer are modeled two-dimensionally. This $2 \mathrm{D}$ assumption is motivated by the fact that the fin height is significantly larger than the xy-dimensions. Also the original 3D thermal diffusion problem in the base plate is simplified to a $2 \mathrm{D}$ problem as the xy-dimensions of the base plate are much larger than its height. The validity of these simplifications is assessed in section 5.2 .2

\subsubsection{Fluid dynamics modeling}

Assuming an incompressible fluid and two-dimensional flow in the xy-plane, the continuity equation and Navier-Stokes equation are defined as:

$$
\begin{array}{r}
\rho_{f}(\nabla \cdot \mathbf{u})=0 \\
\rho_{f} \cdot(\mathbf{u} \cdot \nabla) \mathbf{u}=-\nabla p+\mu_{f}\left(\nabla^{2} \mathbf{u}\right)+\mathbf{F}
\end{array}
$$




\section{D model}

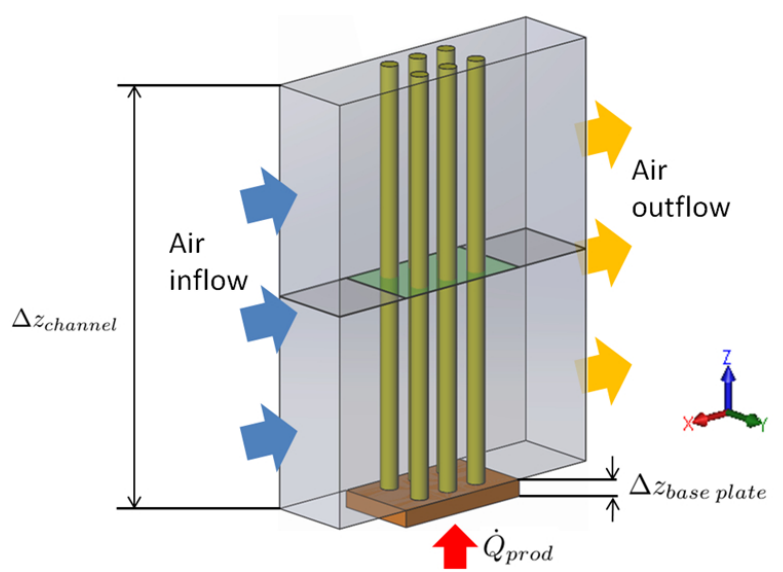

Pseudo 3D model

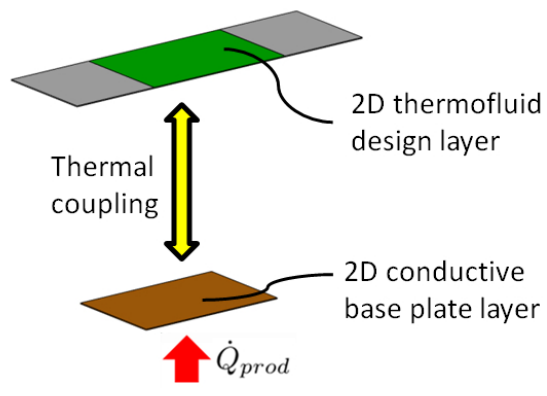

Figure 1: Left: 3D sketch of a forced convection heat sink as treated in this work including base plate (brown), fins (yellow) and modeled domain for air flow (grey). Exemplarily, pin fins are depicted in yellow. Right: Simplified pseudo 3D model consisting of a 2D thermofluid design layer where green corresponds to the design domain and grey to non-optimizable fluid area as well as the $2 \mathrm{D}$ modeled base plate (brown). The thermofluid design layer is also shown in the 3D model for illustrative purposes.

where $\rho_{f}$ is the fluid density, $\mathbf{u}$ is the fluid velocity vector, $p$ is the pressure field, $\mu_{f}$ is the dynamic viscosity of the fluid, and $\mathbf{F}$ is the Brinkman friction term. The Brinkman friction term is used in fluid flow topology optimization to penalize flow through solid areas within the design domain and corresponds to the force exerted on a fluid flowing through an ideal porous medium [37. It is defined as:

$$
\mathbf{F}(\gamma)=-\bar{\alpha} \mathbf{u} I_{\alpha}(\gamma) \quad \text { in } \Omega_{d}
$$

where $\bar{\alpha}$ is the maximum inverse permeability of the porous medium and $I_{1}(\gamma)$ a suitable function for the inverse permeability interpolation which is stated and discussed in section 3.1. The maximum inverse permeability is computed by:

$$
\bar{\alpha}=\mu_{f} /\left(D a L_{c}^{2}\right)
$$

where $D a$ is the Darcy number and $L_{c}$ a characteristic length which corresponds to the design domain width within this work. Ideally, the value of $\bar{\alpha}$ would be set to infinity to prevent any fluid from flowing through solid areas. However, very high values of $\bar{\alpha}$ can cause numerical problems and issues with convergence to poor local optima. Therefore, the choice of $\bar{\alpha}$, or $D a$ from which $\bar{\alpha}$ follows, needs to be a tradeoff that ensures negligible fluid flow through the solid while avoiding problems with numerical stability and the optimization trajectory. Outside the design domain, no Brinkman friction is exerted on the fluid; hence:

$$
\mathbf{F}=\mathbf{0} \quad \text { in } \Omega_{f}
$$


model $a$

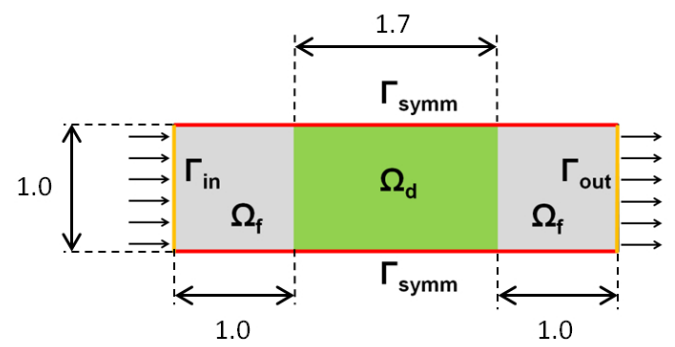

model $b$

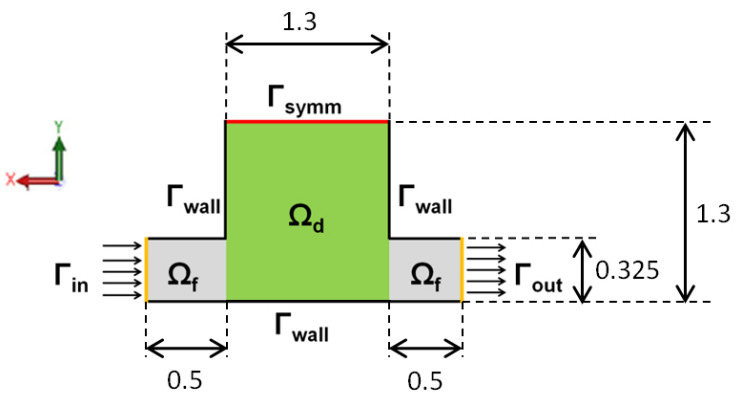

Figure 2: Schematic illustration of the domains $\Omega_{i}$ and boundaries $\Gamma_{j}$ as well as dimensions in mm of heat sink model $a$ and model $b$. The design domain is highlighted in green, the non-optimizable fluid area in grey, the symmetry boundaries are marked with a red line, the inlet and outlet boundaries with an orange line, and the wall boundaries with a black line. Please note that the models are not true to scale relative to each other.

The pressure drop between inlet and outlet, $\Delta p$, is prescribed in both model $a$ and model $b$ :

$$
\left.p\right|_{\Gamma_{\text {in }}}-\left.p\right|_{\Gamma_{\text {out }}}=\Delta p
$$

where $\left.p\right|_{\Gamma_{\text {in }}}$ and $\left.p\right|_{\Gamma_{\text {out }}}$ is the respective pressure at the inlet and outlet of the modeled domain as specified in Fig. 2. Additionally, a normal laminar inflow is set at the inlet boundary, $\Gamma_{i n}$. The symmetry boundary condition for the fluid flow is given by the following two equations:

$$
\begin{array}{rrr}
\mathbf{u} \cdot \mathbf{n}=0 & \text { on } \Gamma_{\text {symm }} \\
\mathbf{K}-(\mathbf{K} \cdot \mathbf{n}) \mathbf{n}=\mathbf{0} & \text { on } \Gamma_{\text {symm }}
\end{array}
$$

where $\mathbf{n}$ is the vector normal to the respective boundary and $\mathbf{K}$ is defined as:

$$
\mathbf{K}=\left[\mu_{f}\left(\nabla \mathbf{u}+(\nabla \mathbf{u})^{T}\right)\right] \mathbf{n}
$$

In model $b$, a no slip condition is imposed on the wall boundaries, $\Gamma_{\text {wall }}$.

\subsubsection{Heat transfer modeling}

In the thermofluid design layer outside the design domain, the $2 \mathrm{D}$ thermal convection-diffusion equation without heat source or heat sink is solved which is given by:

$$
\rho_{f} c_{f} \mathbf{u} \nabla T_{f}-\nabla \cdot\left(k_{f} \nabla T_{f}\right)=0 \quad \text { in } \Omega_{f}
$$

where $T_{f}$ is the temperature field in the thermofluid design layer, $c_{f}$ the specific fluid heat capacity, and $k_{f}$ the thermal conductivity of the fluid. Within the design domain, the following $2 \mathrm{D}$ thermal convection-diffusion equation is solved:

$$
\gamma \rho_{f} c_{f} \mathbf{u} \nabla T_{f}-\nabla \cdot\left(k_{f} I_{k}(\gamma) \nabla T_{f}\right)=\frac{\dot{q}_{\text {inter }}(\gamma)}{\Delta z_{\text {channel }}} \quad \text { in } \Omega_{d}
$$


where $I_{k}(\gamma)$ is a function that interpolates between the thermal conductivity of the fluid, $k_{f}$, and that of the heat sink material, $k_{s}$. The interpolation function used for $I_{k}(\gamma)$ is stated in section 3.1. The term $\dot{q}_{\text {inter }}(\gamma)$ represents the heat transferred from the solid base plate to the thermofluid design layer and $\Delta z_{\text {channel }}$ is the height of the air channel and fins. By multiplying the advective energy transport term in equation (11) with $\gamma$, a linear penalization of the advective energy transport through solid areas is added, as it was done in [25, 28, 31]. This ensures that slight fluid leakage through solid areas, which is to some degree unavoidable in density-based topology optimization, does not result in advective energy transport through these regions. It should be noted that the temperature problem is coupled to the fluid problem through the velocity field $\mathbf{u}$; nevertheless, there is no back coupling from the temperature problem to the fluid problem since constant thermophysical properties for air are assumed throughout this work. The thermophysical properties of air used in this study are stated in section 5.1. In the metal base plate, a 2D heat conduction problem is solved:

$$
\nabla \cdot\left(k_{s} \nabla T_{s}\right)=-\frac{\dot{Q}_{\text {prod }}}{V_{\text {base plate }}}+\frac{\dot{q}_{\text {inter }}(\gamma)}{\Delta z_{\text {base plate }}} \quad \text { in } \Omega_{d}
$$

where $k_{s}$ is the base plate thermal conductivity, $T_{s}$ is the temperature field in the base plate, $\dot{Q}_{\text {prod }}$ is the prescribed heat production rate, $V_{\text {base plate }}$ is the volume of the base plate, and $\Delta z_{\text {base plate }}$ is the height of the base plate. In all studies presented in this work, apart from section 5.2.5, a uniform heat production rate is assumed in the base plate. The out-of-plane heat transfer between base plate and thermofluid design layer, $\dot{q}_{\text {inter }}(\gamma)$, needs to flexibly represent both the conductive heat transfer from the base plate into the heat sink fins as well as the convective heat transfer from the base plate to the fluid. This is achieved by using a heat transfer coefficient which is interpolated between a high conductive heat transfer into the fins and a lower convective heat transfer to the fluid:

$$
\dot{q}_{\text {inter }}(\gamma)=h_{f} I_{h}(\gamma)\left(T_{s}-T_{f}\right)
$$

where $h_{f}$ is the heat transfer coefficient describing the convective heat transfer to the fluid and $I_{h}(\gamma)$ is a function interpolating between $h_{f}$ and the heat transfer coefficient from the base plate into the heat sink fins, $h_{s}$. The parameter $h_{s}$ represents the conductive heat transfer resistance in the z-direction in the fins and is empirically determined.

The fluid inlet temperature is set to the fixed value $T_{i n}$ :

$$
T_{f}=T_{i n} \quad \text { on } \Gamma_{i n}
$$

The outlet, symmetry, and adiabatic wall boundary condition for the temperature field in the thermofluid design layer is given by:

$$
\mathbf{n} \cdot \nabla T_{f}=0 \quad \text { on } \Gamma_{\text {out }} \cup \Gamma_{\text {symm }} \cup \Gamma_{\text {wall }}
$$


Perfect thermal insulation is moreover assumed on all boundaries of the heat sink base plate:

$$
\mathbf{n} \cdot \nabla T_{s}=0 \quad \text { on } \partial \Omega_{d}
$$

\section{Topology optimization}

\subsection{Interpolation functions}

A continuous design field, $\gamma(\mathbf{x})$, taking values between 0 and 1 is introduced in density-based topology optimization. In the given design problem, 1 corresponds to fluid passage, 0 to heat sink fin material, and interpolation functions are used to represent intermediate values. For the Brinkman friction term, an interpolation function as stated in [33] and first introduced in [37] is used in this work:

$$
I_{\alpha}(\gamma)=\frac{1-\gamma}{1+b_{\alpha} \gamma}
$$

where $b_{\alpha}$ is a parameter determining the convexity of the interpolation. For the interpolation of the thermal conductivity within the design layer and the heat transfer between heat sink base plate and thermofluid design layer, a RAMP-style interpolation as used in 33] and originally presented in 38 is applied:

$$
I_{j}(\gamma)=\frac{\gamma\left(C_{j}\left(1+b_{j}\right)-1\right)+1}{C_{j}\left(1+b_{j} \gamma\right)} \quad \mathrm{j}=\mathrm{k}, \mathrm{h}
$$

where $b_{j}$ is the interpolation convexity parameter and $C_{j}$ is defined in the respective case by:

$$
\begin{aligned}
C_{k} & =\frac{k_{f}}{k_{s}} \\
C_{h} & =\frac{h_{f}}{h_{s}}
\end{aligned}
$$

\subsection{Problem formulation}

The optimization objective considered in this work is to minimize the heat sink thermal resistance for a prescribed pressure drop over the heat sink and prescribed heat production rate. The thermal resistance of the heat sink, $R_{t h}$, is defined as:

$$
R_{t h}(\gamma, \mathbf{s})=\frac{T_{s, a v}(\gamma, \mathbf{s})-T_{i n}}{\dot{Q}_{\text {prod }}}
$$

where $T_{s, a v}$ is the average temperature in the base plate and $\mathbf{s}$ is the state vector of the thermofluid problem stated in section 2.2 . Thus, the topology optimization problem can be stated as follows:

$$
\begin{array}{lll}
\min _{\gamma} .: & R_{t h}(\gamma, \mathbf{s}) \\
\text { s.t.: } & r(\gamma, \mathbf{s})=0 \\
& \iint_{\Omega_{d}}(1-\gamma) d x d y-f A_{\Omega_{d}} \leq 0 & \\
& 0 \leq \gamma(\mathbf{x}) \leq 1 \quad \forall \mathbf{x} \in \Omega_{d}
\end{array}
$$


where $r(\gamma, \mathbf{s})$ is the residual of the finite element formulation of the thermofluid problem stated in section 2.2. $A_{\Omega_{d}}$ is the design domain area, and $f$ is the prescribed maximum fin volume fraction or fin cross-sectional area fraction. The pressure drop over the heat sink does not appear explicitly as constraint in this statement since it is prescribed as boundary condition in the thermofluid model.

$$
\begin{array}{lll}
\min _{\gamma} .: & \Delta p(\gamma, \mathbf{s}) \\
\text { s.t.: } & r(\gamma, \mathbf{s})=0 & \\
& \iint_{\Omega_{d}}(1-\gamma) d x d y-f A_{\Omega_{d}} \geq 0 \quad & \\
& 0 \leq \gamma(\mathbf{x}) \leq 1 \quad \forall \mathbf{x} \in \Omega_{d}
\end{array}
$$

In the case of pressure drop minimization, a minimum cross-sectional area fraction constraint is needed since the lowest pressure drop for a prescribed fluid inlet velocity will always be obtained by a design with no fin material in the cross section $(f=0)$. It should be noted that in the thermal resistance minimization problem, in contrast, a maximum cross-sectional area fraction constraint is applied as the values of $f$ analyzed in section 5.2.4 are below the $f$ value that minimizes the heat sink thermal resistance in that specific case.

\section{3. $P D E$ filter and projection}

Density filtering is needed in thermofluid topology optimization to avoid problems with illposedness of the optimization problem [39. A Helmholtz-type partial differential equation (PDE) filter [40] is used in this study which is defined as follows:

$$
-r_{\text {filter }}^{2} \nabla^{2} \tilde{\gamma}+\tilde{\gamma}=\gamma \quad \text { in } \Omega_{d}
$$


where $\tilde{\gamma}$ is the filtered design field and $r_{\text {filter }}$ is the filter parameter. The following boundary conditions are used for the filter PDE:

$$
\begin{array}{rr}
\tilde{\gamma}=1 & \text { on } \partial \Omega_{d} \backslash\left(\Gamma_{\text {symm }} \cup \Gamma_{\text {wall }}\right) \\
\mathbf{n} \cdot \nabla \tilde{\gamma}=0 & \text { on } \Gamma_{\text {symm }} \cup \Gamma_{\text {wall }}
\end{array}
$$

To reduce the gray area between solid and fluid which is inherently introduced by the filtering process, a smoothed heaviside projection [4] is applied on the filtered design field:

$$
\overline{\tilde{\gamma}}=\frac{\tanh (\beta \eta)+\tanh (\beta(\tilde{\gamma}-\eta))}{\tanh (\beta \eta)+\tanh (\beta(1-\eta))}
$$

where $\overline{\tilde{\gamma}}$ is the projected design field, $\beta$ is a parameter controlling the projection steepness, and $\eta$ is the projection threshold parameter. It should be noted that the projected design field becomes the physical meaningful one in the interpolation functions (Eq. 117 ) and (18)) and the thermal convection-diffusion equation (Eq. (11)).

\section{Implementation}

The heat sink topology optimization models are implemented in the commercial finite element software COMSOL Multiphysics [42. The fluid problem (Eq. (1) and (2)) is solved with COMSOL's CFD Module using a second order discretization for the velocity field and first order discretization for the pressure. COMSOL's Heat Transfer Module is used to solve the thermal convection-diffusion equation (Eq. (10) and (11)) and the thermal diffusion problem in the base plate (Eq. 12) using second order elements for $T_{f}$ due to the steep temperature gradients in the thermofluid design layer and first order elements for $T_{s}$. The filter PDE (Eq. (24)) is implemented in the Coefficient Form PDE Interface using a linear discretization and the filter parameter is set to 1.5 times the maximum element size in the design domain. Triangular elements are used for all PDEs and no stabilization scheme is applied as it was found to slightly blur the sensitivities within COMSOL's optimizer when compared to a finite difference check. The parallel sparse direct solver PARDISO [43 that is available in COMSOL is used to solve the system of discretized finite element equations and segregated solver steps are used for the fluid problem, thermal problem, and filter PDE. The optimization is conducted within COMSOL's Optimization Module which automatically solves the adjoint problem to provide sensitivities for the objective and constraint functionals and the globally convergent version of the Method of Moving Asymptotes (GCMMA) [44] is used as the optimization method. A continuation approach [39, 45] is applied on the convexity parameters of the interpolation functions, $b_{\alpha}, b_{h}, b_{k}$, and the steepness parameter of the design projection, $\beta$. This is done to ensure a more convex optimization problem in the beginning and to consequently gradually increase the penalization of intermediate densities 
as well as to increase the sharpness of the solid-fluid interface. The choice of the final values

240 important as they mainly determine whether there will be areas with unphysical intermediate densities in the final design. This is due to the high conductivity difference between heat sink metal and air which is used as coolant in this work. This high conductivity difference can make thin features with intermediate densities that have increased thermal conductivity compared to air but for specific conditions would allow for higher fluid velocities, larger model dimensions, and higher resolution of the problem at the cost of longer development time.

Table 1: Values of optimization parameters and number of model evaluations during the continuation approach for model $a$ and model $b$.

\begin{tabular}{lllllll}
\hline Continuation step & $n_{\text {eval,model a }}$ & $n_{\text {eval,model } b}$ & $q_{\alpha}$ & $q_{k}$ & $q_{h}$ & $\beta$ \\
\hline 1 & 500 & 600 & 8 & 0.1 & 0.1 & 1 \\
2 & 250 & 300 & 4 & 5 & 2 & 1.5 \\
3 & 150 & 150 & 2 & 20 & 8 & 2 \\
4 & 100 & 100 & 1 & 50 & 8 & 2.5 \\
$5,6,7,8,9$ & 80 & 80 & 1 & 50 & 8 & $3,4,5,6,7$ \\
10 & 80 & 80 & 1 & 50 & 8 & 7 \\
\hline
\end{tabular}


Table 2: Values of constant optimization and mesh parameters for model a and model $b$.

\begin{tabular}{lll}
\hline Parameter & Model a value & Model $b$ value \\
\hline$D a[-]$ & $10^{-5}$ & $10^{-5}$ \\
max. element size in $\Omega_{d}[\mathrm{~mm}]$ & $1.33 \times 10^{-2}$ & $8.66 \times 10^{-3}$ \\
max. element size in $\Omega_{a}[\mathrm{~mm}]$ & $2.86 \times 10^{-2}$ & $1.63 \times 10^{-2}$ \\
$r_{\text {filter }}[\mathrm{mm}]$ & $2.00 \times 10^{-2}$ & $1.30 \times 10^{-2}$ \\
\hline
\end{tabular}

\section{Results and discussion}

\subsection{Problem setup}

Within this work, the thermophysical properties of air are assumed constant and evaluated at $20{ }^{\circ} \mathrm{C}$. The resulting values are given in Table 3 A thermal conductivity of $400 \mathrm{~W} /(\mathrm{m} \mathrm{K})$, which corresponds to the conductivity of copper, is assumed in the heat sink base plate. The high conductivity difference between copper and air can cause issues with regards to grey area in the final design and numerical stability during the optimization as discussed in section 4 For this reason, the value of $C_{k}$, which determines the solid conductivity in the xy-plane in the thermofluid design layer, is in both models set to lower thermal conductivities than the thermal conductivity assumed in the heat sink base plate. The chosen $C_{k}$ value corresponds to a solid thermal conductivity of $24 \mathrm{~W} /(\mathrm{m} \mathrm{K})$ in model $a$ and $0.072 \mathrm{~W} /(\mathrm{m} \mathrm{K})$ in model $b$ which makes the latter a more academic model. It should be noted that the $C_{k}$ value chosen in model $b$, which is lower than the value used in model $a$ is not chosen due to problems with grey area or numerical stability during the optimization but because of convergence to only locally optimal topologies that were observed during preliminary model $b$ optimizations. This issue is further discussed in section 5.3 In the model a pseudo 3D and full 3D validation models, the thermal conductivity of the heat sink base plate is used in all solid domains to assess the influence of the chosen lower value of $C_{k}$ in the optimization model. The abovementioned and other constant model parameters used within this work are stated in Table 4. As already stated in section 2.2.2, a uniform heat production rate is assumed in the heat sink base plate in all studies presented in this work apart from the model presented in section 5.2.5 where hotspots are studied. Moreover, the base plate thickness differs in section 5.2 .5 from the value given in Table 4 .

\subsection{Results model a}

Thermofluid topology optimization problems are quite non-convex and can easily converge to only locally optimal topologies depending on the starting guess or initial design. Therefore, several initial designs are used for each optimization in this work and only the respective best performing design is presented. The initial designs used for the optimization of model $a$ are shown in Fig. 3 . 
Table 3: Constant thermophysical properties of air used in this work.

\begin{tabular}{lr}
\hline Thermophysical property & \multicolumn{1}{c}{ Value } \\
\hline$c_{\text {air }}[\mathrm{J} /(\mathrm{kg} \mathrm{K})]$ & 1006 \\
$k_{\text {air }}[\mathrm{W} /(\mathrm{m} \mathrm{K})]$ & 0.024 \\
$\mu_{\text {air }}[\mathrm{Pa} \mathrm{s}]$ & $1.94 \times 10^{-5}$ \\
$\rho_{\text {air }}\left[\mathrm{kg} / \mathrm{m}^{3}\right]$ & 1.204 \\
\hline
\end{tabular}

Table 4: Constant parameters used in the heat sink model $a$ and model $b$.

\begin{tabular}{lll}
\hline Parameter & Model a value & Model $b$ value \\
\hline$C_{k}[-]$ & $1 / 1000$ & $1 / 3$ \\
$h_{f}\left[\mathrm{~W} /\left(\mathrm{m}^{2} \mathrm{~K}\right)\right]$ & 50 & 50 \\
$h_{s}\left[\mathrm{~W} /\left(\mathrm{m}^{2} \mathrm{~K}\right)\right]$ & $2 \times 10^{5}$ & $2 \times 10^{5}$ \\
$k_{s, \text { base plate }}[\mathrm{W} /(\mathrm{m} \mathrm{K})]$ & 400 & 400 \\
$\dot{Q}_{\text {prod }}[\mathrm{W}]$ & 0.175 & 0.25 \\
$T_{\text {in }}\left[{ }^{\circ} \mathrm{C}\right]$ & 20 & 20 \\
$\Delta z_{\text {base plate }}[\mathrm{mm}]$ & 0.2 & 0.2 \\
$\Delta z_{\text {channel }}[\mathrm{mm}]$ & 8 & 8 \\
\hline
\end{tabular}

For all model a optimizations presented in this work, the initial designs (a) - (k) and a uniform initial density field of value 0.8 are used. The initial designs (a) - (c) can be interpreted as parallel fin heat sink when the periodic structure is considered. The asymmetry of the designs (d) - (k) with regards to a horizontal line in the center of the design domain is intended as this was found

to yield improved optimization results compared to designs that are symmetric with regards to this line. Note that only the initial designs (g), (h), and (i) led to best performing final designs in all optimizations conducted for this work; all other initial guesses led to only locally optimal designs. Still, it is important to test various initial topologies as it is not known a priori which one will yield the best performing final design in the different optimizations.

\subsubsection{Analysis and discussion of optimized designs}

An exemplary model a optimization result for a pressure drop over the heat sink of $3 \mathrm{~Pa}$ with corresponding velocity magnitude field, temperature field in the thermofluid design layer, and temperature field in the metal base plate is shown in Fig. 4. Four fins are formed within the design domain where the bottom left and top right fin are halved by the symmetry boundary. The two fins in the bottom part of the design domain reach the left boundary and the two fins in the upper part of the design domain reach the right boundary so that the fins are almost arranged diagonally from the bottom left to the top right of the design domain. All fins have to some degree 


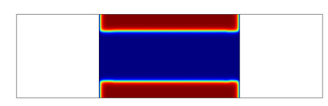

(a)

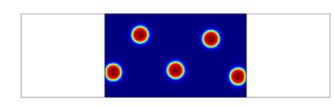

(e)

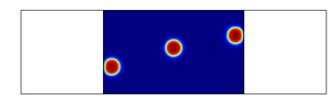

(i)

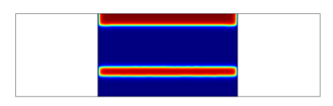

(b)

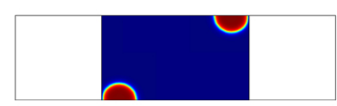

(f)

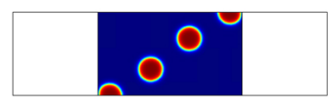

(j)

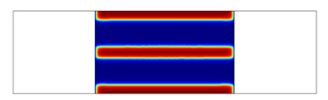

(c)

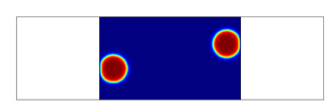

(g)

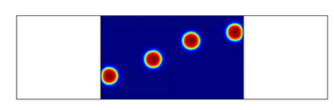

(k)

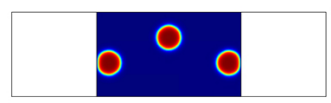

(d)

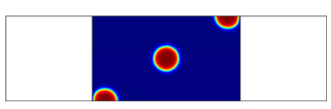

(h)

Figure 3: Initial designs used for model a (red is solid and blue is fluid). The designs (a) - (k) as well as a uniform initial density field of value 0.8 are used to generate the model a results presented in this study.

a streamlined shape except for the bottom left fin which is almost triangular. This fin is probably shaped in this way to fit closely to the left boundary of the design domain and a more streamlined the metal base plate. A very uniform temperature can be seen in the base plate with a maximum temperature difference in the plate of slightly less than $0.4 \mathrm{~K}$.

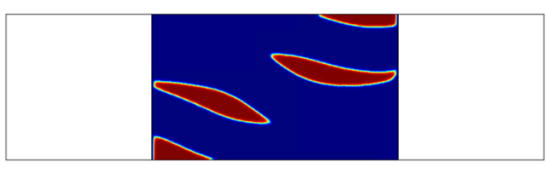

Design

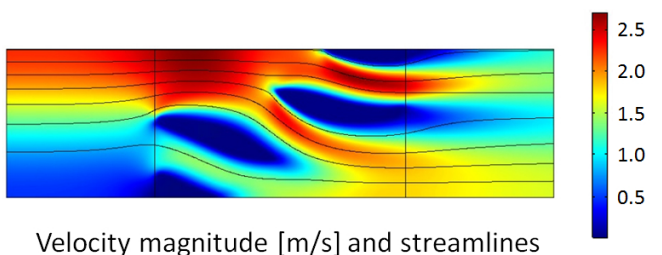

Velocity magnitude $[\mathrm{m} / \mathrm{s}]$ and streamlines

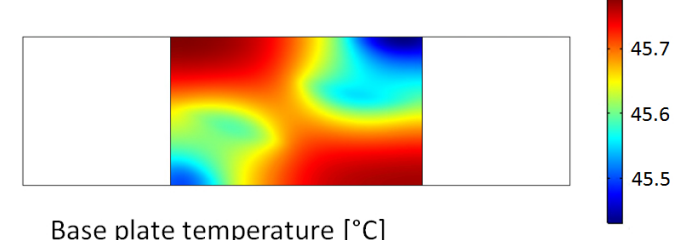

Figure 4: Exemplary model a design obtained for a pressure drop over the heat sink of 3 Pa where red corresponds to solid and blue to fluid. Additionally, the corresponding velocity field, temperature field in the thermofluid design layer, and temperature field in the metal base plate are shown. 
Figure 5 shows the influence of the prescribed pressure drop over the heat sink on the optimized

320 thermofluid topology optimization.

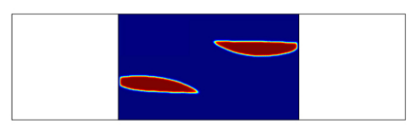

(a)

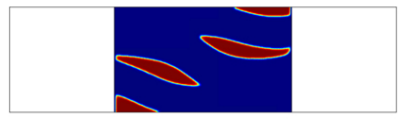

(d)

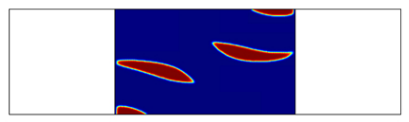

(b)

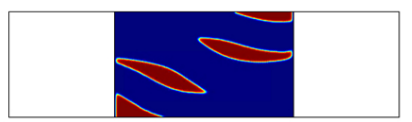

(e)

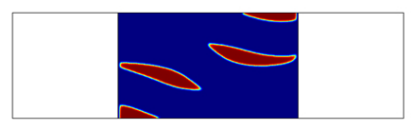

(c)

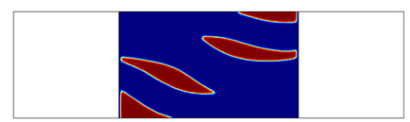

(f)

Figure 5: Influence of prescribed pressure drop over heat sink on the optimized topology for model a. The pressure drop takes the values 0.5 (a), 1 (b), 2 (c), 3 (d), 5 (e), and $7 \mathrm{~Pa}$ (f). 


\subsubsection{Model validation and parametric studies}

It is important to verify the topology optimization model for several reasons: The accuracy of

345 which can be done by replacing this modeling approach with an explicit separate modeling of solid and fluid domains and a body-fitted mesh resolving the physics in the thermal boundary layer with high accuracy. Moreover, the threshold value for the explicit solid fluid boundary representation, $\overline{\tilde{\gamma}}=0.8$, and the assumption of a lower solid conductivity in the thermofluid design layer plane, i.e. the choice of $C_{k}$, should be validated. The influence of these factors can be checked in a pseudo $3 \mathrm{D}$ validation model which will subsequently be referred to as the $2 \mathrm{D}$ validation model. Moreover, a full 3D validation model is used to quantify the influence of the assumptions inherent to the pseudo 3D modeling approach. These are the simplification of a 3D thermofluid problem to the 2D thermofluid design layer and the interpolation of the simplified heat transfer coupling between the $2 \mathrm{D}$ base plate model and the thermofluid design layer, i.e. the choice of the parameters $h_{f}$ and $h_{s}$. These effects are captured explicitly in the 3D model as convective heat transfer from the base plate to the fluid and conductive heat transport from the base plate into the heat sink fins. Furthermore, the drag force from the top and bottom model boundary on the air flow is captured in the $3 \mathrm{D}$ model while it is not considered in the $2 \mathrm{D}$ approach.

Figure 6 shows the 3D boundary layer mesh for the optimized design shown in Fig. 4 and the corresponding 3D velocity magnitude field and temperature field. The mesh consists of approximately 403,000 elements in total and is shown on the fin and base plate surface as well as on the bottom wall and left symmetry boundary of the channel. The $2 \mathrm{D}$ assumption in the optimization model seems to be well justified for the fluid problem as the flow shows very little variation along the z-coordinate apart from a small hydraulic boundary layer near the top and bottom walls. The z-dependency of the temperature field is slightly more pronounced as there is a temperature drop of around $8{ }^{\circ} \mathrm{C}$ along the fins in addition to a thermal boundary layer above the base plate. Nevertheless, the $2 \mathrm{D}$ thermofluid design layer assumption seems to be justified as an approximation. The temperature drop in the fins of around $8{ }^{\circ} \mathrm{C}$ predicted by the $3 \mathrm{D}$ model agrees well with the around $4{ }^{\circ} \mathrm{C}$ temperature difference between the fins in the thermofluid design layer and the base plate which can be seen in the corresponding pseudo 3D model results shown in Fig. 4 as the fin temperature in the pseudo 3D model can be interpreted as an averaged temperature along the height of the fins. Hence, the value of the pseudo 3D model parameter $h_{s}$, which represents the conductive thermal resistance along the z-coordinate in the fins, yields good results for the presented case.

To provide a more quantitative means of evaluating the agreement between the optimization model and the 2D and 3D validation model, the heat sink thermal resistance and Reynolds number are compared over a pressure drop range from $0.5 \mathrm{~Pa}$ to $7 \mathrm{~Pa}$ which is shown in Fig. 7. Note 

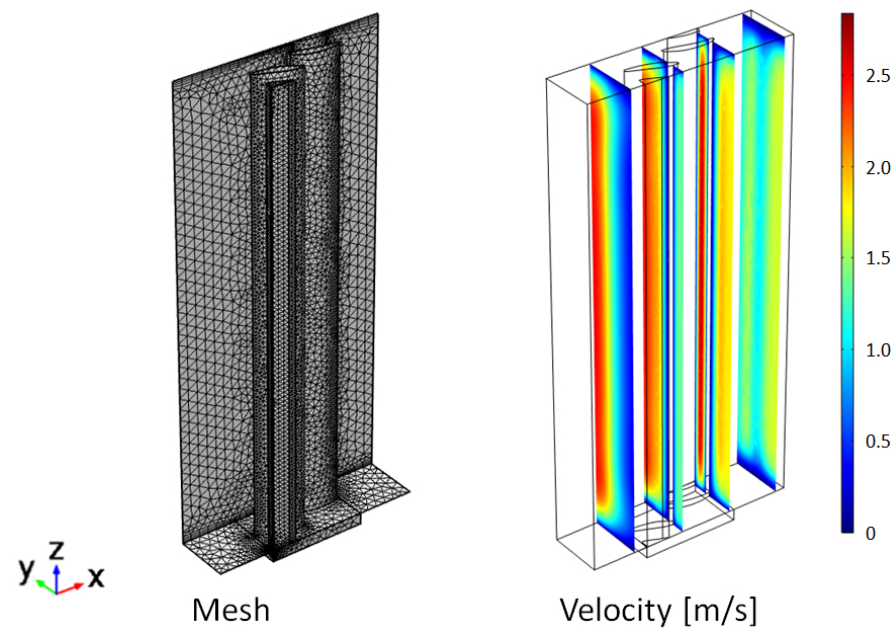

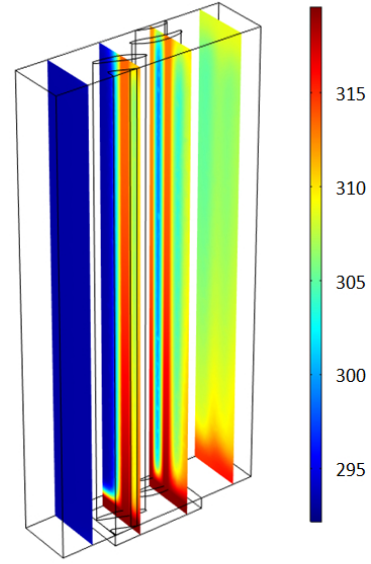

Temperature $\left[{ }^{\circ} \mathrm{C}\right]$

Figure 6: Mesh used in the 3D body-fitted mesh validation model of the optimized model a geometry shown in Fig. 4 with corresponding velocity and temperature field.

that all quantitative model a results presented in the following are obtained by evaluating the experimental validation of the flow conditions should be conducted which is left for future works 2. The optimization model and 2D validation model show good agreement in terms of predicted

\footnotetext{
${ }^{2}$ As mentioned in the introduction and shown in [16, fabrication of topology optimized designs using additive manufacturing techniques may have great potential due to the design freedom that these methods provide. How-
} 
Reynolds number where the $2 \mathrm{D}$ validation model consistently predicts slightly lower values. This

Cross-checking the optimization results is important as poor local optima within the generated designs can be identified. Nevertheless, it is limited in the sense that the designs are only compared

ever, it should be noted that the dimensions of the design domains treated in this work are in the millimeter to submillimeter range which are presently more suited to micromachining or conventional chemical etching techniques than most mature additive manufacturing techniques. These dimensions were chosen due to the limitation to low Reynolds numbers of the COMSOL modeling framework. Achieving low Reynolds numbers in models with larger model dimensions would have only been possible at the trade-off of unrealistically low fluid velocities. Generally, treatment of higher Reynolds number problems and larger model dimensions is possible in more customizable and computationally efficient frameworks than COMSOL. 

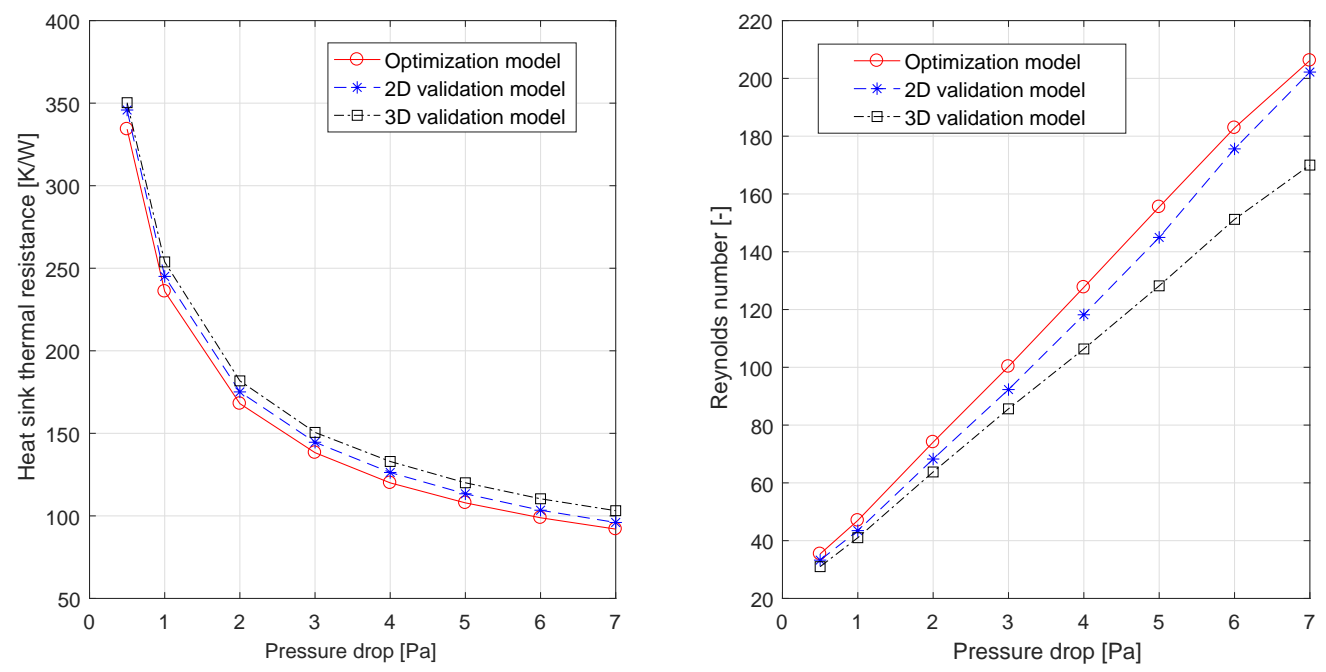

Figure 7: Influence of pressure drop over the heat sink on the heat sink heat transfer resistance as defined in equation 21] (left) and the Reynolds number of the optimized designs (right). Results are shown for the model a optimization model, the corresponding $2 \mathrm{D}$ body-fitted mesh validation model, and 3D body-fitted mesh validation model.

Table 5: Cross-check of model a optimized designs heat transfer resistance [K/W] based on the 2D body-fitted mesh validation model.

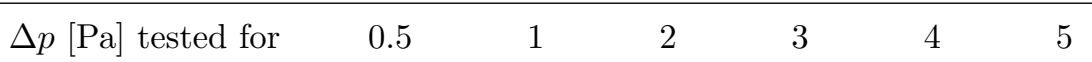

Design for $\Delta p[\mathrm{~Pa}]$

\begin{tabular}{rrrrrrr}
\hline & & & & & & \\
\\
0.5 & $\mathbf{3 4 5 . 8}$ & 260.3 & 205.3 & 180.5 & 165.1 & 154.3 \\
1 & 375.5 & $\mathbf{2 4 5 . 1}$ & 180.8 & 155.9 & 141.2 & 131.0 \\
2 & 439.2 & 257.8 & $\mathbf{1 7 5 . 1}$ & 146.4 & 130.3 & 119.4 \\
3 & 494.1 & 277.0 & 177.6 & $\mathbf{1 4 4 . 6}$ & 126.8 & 115.0 \\
4 & 538.5 & 294.7 & 182.2 & 145.5 & $\mathbf{1 2 6 . 2}$ & 113.7 \\
5 & 595.9 & 319.8 & 191.1 & 149.1 & 127.5 & $\mathbf{1 1 3 . 6}$ \\
\hline
\end{tabular}

within the optimized set and, therefore, it is of interest to benchmark the performance of the topology optimized designs to a conventional heat sink geometry. In this case, a size-optimized parallel fin design is chosen as the reference geometry. All fins have the same thickness, $t h_{f i n}$, which is the optimization variable. Optimal fin thicknesses are determined exemplarily for a pressure drop of $2 \mathrm{~Pa}$ and for 1, 1.5, 2, and 2.5 fins within the design domain. The fins are spaced relative to each other such that the resulting periodic structure has a uniform distance between the fins. The size optimization is conducted for a projection steepness parameter $\beta=7$ to allow

for a fair comparison to the topology optimization as beta $=7$ is the last value of the continuation 
scheme used in this work. Table 6 shows the optimal fin thickness for the different numbers of fins within the design domain and the respective thermal resistance. Moreover, the thermal resistance of the corresponding topology optimized design is shown. It can be seen that the optimal fin thickness decreases with increasing number of fins within the modeled domain as expected. The design with two fins performs best among the size optimized designs having a thermal resistance of $184.5 \mathrm{~K} / \mathrm{W}$ for the optimal fin thickness of $0.06 \mathrm{~mm}$. Still, the topology optimized design for the same pressure drop has a lower thermal resistance of $168.0 \mathrm{~K} / \mathrm{W}$ which is a relative improvement of $9.8 \%$ compared to the best size optimized design.

Table 6: Thermal resistance of the size optimized parallel fin heat sink designs and topology optimized (TO) design for a pressure drop of $2 \mathrm{~Pa}$. Furthermore, the optimal fin thickness is stated for the parallel fin designs.

\begin{tabular}{rrr}
\hline Design & optimal $t h_{\text {fin }}[\mathrm{mm}]$ & $R_{t h}[\mathrm{~K} / \mathrm{W}]$ \\
\hline 1 fin & 0.23 & 250.6 \\
1.5 fin & 0.11 & 189.6 \\
2 fin & 0.06 & $\mathbf{1 8 4 . 5}$ \\
2.5 fin & 0.05 & 218.3 \\
TO design & - & $\mathbf{1 6 8 . 0}$ \\
\hline
\end{tabular}

Figure 8 shows the best performing size optimized design and the topology optimized design to which it is compared. It can be seen that the fins of the topology optimized design are not as thin as the fins of the size optimized design which is advantageous for mechanical stability. Moreover, the topology optimized design may be more robust with regards to manufacturing uncertainty as the performance of parallel fin heat sinks and heat exchangers is sensitive to flow maldistribution caused by small differences of geometry between the channels [51. However, these aspects need to be assessed and quantified in future works. A second exemplary benchmarking of a topology optimized design to a size optimized parallel fin design is presented in section 5.2 .5 for a non-uniform heat generation rate in the base plate.

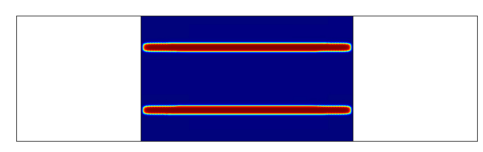

(a)

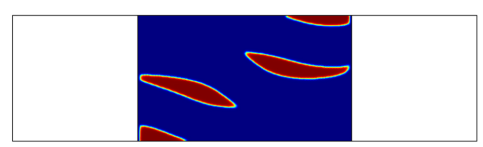

(b)

Figure 8: Size optimized 2 fin design (a) and topology optimized design (b) for a pressure drop of 2 Pa.

\subsubsection{Comparison to pressure drop minimization}

The optimized heat sink fins depicted in Fig. 5 have a relatively streamlined shape as already mentioned above. Therefore, it is of interest to compare the shape of fins optimized with regard to 
thermal resistance minimization and fins of the same cross-sectional area optimized with regard to pressure drop minimization. For a better comparison of the shapes, the same initial design which is generated with thermal resistance minimization objective without volume constraint is used for all optimizations within this section. This design is then used to initialize thermal resistance and pressure drop optimizations with active volume constraint that can be directly compared to each other. Moreover, the air inlet velocity in all pressure drop minimizations is set such that the Reynolds number is the same as in the thermal resistance minimization design without a volume constraint. This initial design and the designs optimized with volume constraints between $19.3 \%$ and $5 \%$ are shown in Fig. 9 The design obtained by thermal resistance minimization without volume constraint consists of a single thin fin stretching across the entire length of the design domain which could be interpreted as a plate fin heat exchanger when considering the periodic structure. The fin is slightly streamlined with a blunt front and rear end where the fin reaches the respective end of the design domain. A similarly shaped fin stretching over the entire length of the design domain is generated for $f=15 \%$ and $f=10 \%$; however, it is becoming increasingly thinner. For $f=5 \%$, two very thin fins with slightly streamlined front and rear end are formed at the position of the single longer fin in the previous designs. The design obtained by pressure drop minimization for $f=19.3 \%$ stretches over the entire length of the design domain as the thermal resistance minimized design at the same $f$ value. However, the pressure drop minimized design resembles more a mix of flat ellipse and NACA shape apart from the blunt front and rear end where the fin reaches the respective end of the design domain. The fin shape is the same for decreasing $f$ values but its length decreases with decreasing $f$ so that the fins do not have a blunt ends for $f=15 \%$ and lower. The comparable problem of optimal 2D cross-sections for drag minimization in laminar Navier-Stokes flow has been treated in the literature by using variational methods of optimal control [52, by numerical solution of the 2D Navier-Stokes and adjoint problem where the latter provides first and second order necessary conditions for a shape with minimal drag [53], and using density-based topology optimization in a finite element framework [54]. Pironneau [52. concludes that the optimal shape has a $90^{\circ}$ wedge shape at the front end and [53] present slender, close to ellipsoid optimal profiles with wedge shaped front and rear end for Reynolds numbers between 1 and 40. Kondoh et al. [54] study the problem for Reynolds numbers of up to 2000 finding similar optimized profiles as 53 for lower Reynolds numbers and, for higher laminar Reynolds numbers, shapes very similar to the pressure drop minimized designs with $f \leq 15 \%$ depicted in Fig. 9. A smoother front and rear end of the profiles is the main difference to the more wedge shaped front and rear ends presented in [54. The smoother shapes obtained in this study are most probably due to the density filtering that smears out fine features which was not applied in [54.

The objective function values for thermal resistance and pressure drop minimization of the 


\section{$R_{\text {th }} \min$}

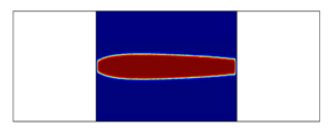

$f=19.3 \%$

(unconstrained)

\section{$\Delta p \min$}

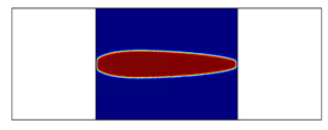

$f=19.3 \%$

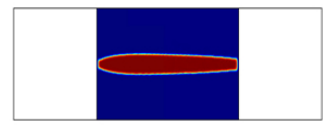

$f=15 \%$

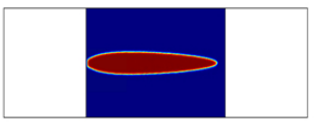

$f=15 \%$

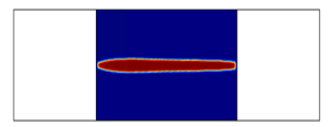

$f=10 \%$

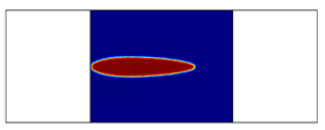

$f=10 \%$

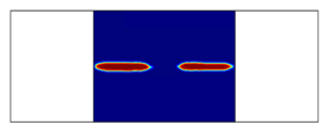

$f=5 \%$

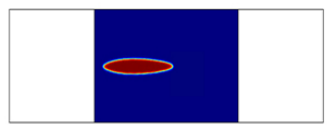

$f=5 \%$

Figure 9: Comparison of thermal resistance minimization and pressure drop minimization for model $a$. The top left design is generated without volume constraint and used as initial design for all other optimizations shown in this figure which are conducted with active constraint on the fraction of solid volume in the design domain.

designs shown in Fig. 9 are given in Table 7. Considering the thermal resistance objective, it can be seen that the thermal resistance increases with decreasing volume constraint values from $225 \mathrm{~K} / \mathrm{W}$ at $f=19.3 \%$ to $282 \mathrm{~K} / \mathrm{W}$ at $f=5 \%$ as the cross-sectional area for heat transfer

from the base plate into the fin and the contact area between fin and airflow decrease. An even stronger increase in thermal resistance with decreasing volume constraint values from from 226 $\mathrm{K} / \mathrm{W}$ at $f=19.3 \%$ to $387 \mathrm{~K} / \mathrm{W}$ at $f=5 \%$ occurs for the pressure drop minimization objective as these designs have less contact area between fin and fluid as those of the thermal resistance minimization for the same $f$ value. The pressure drop decreases significantly with decreasing $f$ for the pressure drop minimization from $2.95 \mathrm{~Pa}$ at $f=19.3 \%$ to $1.28 \mathrm{~Pa}$ at $f=5 \%$. Comparing both optimization objectives for the same $f$, it can be seen that the differences both in thermal resistance and pressure drop increase with decreasing $f$. For $f=19.3 \%$, the relative difference in thermal resistance is $0.3 \%$ and the relative difference in pressure drop $1.7 \%$. However, much larger differences would be observed when comparing designs where several fins are advantageous from 505 a thermal point of view as a single fin is always advantageous for the pressure drop minimization objective.

\subsubsection{Non-uniform heat production rate in the base plate}

In the studies presented above, a uniform heat production rate in the heat sink base plate is considered and a thermal conductivity of $400 \mathrm{~W} /(\mathrm{m} \mathrm{K})$ is assumed in the base plate which

is at the higher side of the thermal conductivities of metals and alloys commonly used in heat sinks. Moreover, air is considered as the coolant which limits the heat flux magnitude that can be rejected by the heat sink compared to other coolants as, for example, water. These factors lead to relatively uniform base plate temperatures that could be well approximated using a lumped 
Table 7: Comparison of thermal resistance minimization and pressure drop minimization objective function values for different volume constraints using model a.

\begin{tabular}{rrrrrrr}
\hline & \multicolumn{5}{c}{$\mathbf{R}_{\text {th }}$ min. objective } & \multicolumn{4}{c}{$\Delta$ m min. objective } \\
$f[\%]$ & $R_{t h}[\mathrm{~K} / \mathrm{W}]$ & $\Delta p[\mathrm{~Pa}]$ & $R e[-]$ & $R_{t h}[\mathrm{~K} / \mathrm{W}]$ & $\Delta p[\mathrm{~Pa}]$ & $R e[-]$ \\
\hline & & & & & & \\
19.3 & 225.4 & 3.00 & 115 & 226.1 & 2.95 & 115 \\
15 & 226.5 & 3.00 & 131 & 244.0 & 2.45 & 115 \\
10 & 234.6 & 3.00 & 152 & 289.3 & 1.87 & 115 \\
5 & 282.0 & 3.00 & 173 & 387.3 & 1.28 & 115 \\
\hline
\end{tabular}

model for the base plate, i.e. relying on a pure 2D optimization model instead of the presented pseudo 3D approach which captures the thermal diffusion problem in the base plate. Therefore, a model with non-uniform heat production rate in the base plate and reduced base plate thickness that leads to thermal hotspots is introduced in this section to demonstrate the added value of the pseudo 3D optimization model. The base plate thickness considered in this section is 0.005 $\mathrm{mm}$ and the same total heat production in the base plate as in the studies above is assumed; however, the heat is only released in one circular area in the bottom left and one circular area in the top right part of the design domain. An exemplary topology optimization using this model is conducted for a pressure drop of $2 \mathrm{~Pa}$ and the optimized design and the corresponding velocity magnitude, design layer temperature, and base plate temperature are shown in Fig. 10. The areas where the heat generation in the base plate occurs are marked with yellow circles. The resulting design consists of three fins which is a different topology than the corresponding $2 \mathrm{~Pa}$ design for a uniform base plate heat production rate (see Fig. 5f) that comprises 4 fins. Moreover, an optimization for this pressure drop is conducted for comparison using an optimization model with a lumped model for the heat sink base plate, i.e. assuming a spatially non-varying base plate temperature. This optimization results in a topology comprising 4 fins similar to the design shown in Fig. 5f which is not surprising as the base plate temperature only varies by around $0.4^{\circ} \mathrm{C}$ in the case of uniform heat generation. In the case of non-uniform heat generation in the base plate, the topology with three fins is advantageous since the top and bottom fins can be placed directly on the respective area of heat generation and hence effectively dissipate the heat from there. For this reason, the bottom and top fin have an around $10{ }^{\circ} \mathrm{C}$ higher temperature than the middle fin. The highest temperatures in the base plate occur around the areas of heat generation even though fins are placed above these areas to remove the heat. A temperature difference of $15^{\circ} \mathrm{C}$ occurs between the hotspots and the coldest part of the base plate which is below the middle fin. Comparing the heat sink thermal resistances realized by the different optimization models, it is interesting to note that the non-uniform heat generation design has a thermal resistance of 164.6 


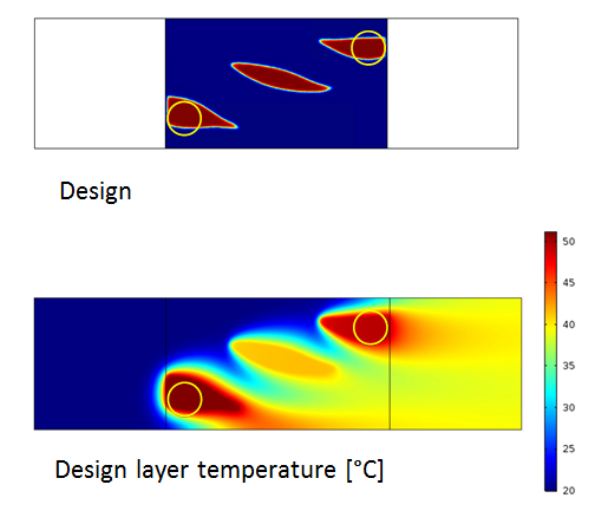

$\mathrm{K} / \mathrm{W}$ which is slightly lower than the thermal resistance of $168.0 \mathrm{~K} / \mathrm{W}$ realized by the uniform heat generation model and $167.3 \mathrm{~K} / \mathrm{W}$ realized by the lumped base plate model. This shows that the topology optimization can make use of the increased hotspot temperatures and remove the heat more efficiently than in the case of an isothermal or almost isothermal base plate which provides some confirmation of the usefulness of explicitly modeling the thermal diffusion in the base plate in the optimization model.

Figure 10: Topology optimized model a design obtained for a non-uniform heat production rate in the base plate and a pressure drop over the heat sink of $2 \mathrm{~Pa}$. Red corresponds to solid, blue to fluid and the areas where the heat input into the base plate occurs are marked by yellow circles. Additionally, the corresponding velocity field, temperature field in the thermofluid design layer, and temperature field in the metal base plate are shown.

As presented in section 5.2 .3 for the case of uniform heat generation in the base plate, a comparison to a size optimized parallel heat sink is conducted. Also in this case 1 fin, 1.5 fins, 2 fins, and 2.5 fins within the design domain, spaced such that the fins have a uniform distance in the periodic structure, are considered. Furthermore, a design with with 2 fins is optimized where the fins are spaced such that the fin center coincides with the center of the circular areas of heat input which results in a slightly irregular fin pattern when considering the periodic fin structure. For each of these cases, the optimal fin thickness is determined and the resulting fin thickness and thermal resistances of these size optimized designs and the topology optimized design are provided in Table 8 . The design 2 fin (a) refers to the design with 2 fins and a uniform fin spacing in the periodic structure and the design 2 fin (b) refers to the design with 2 fins where the center of the respective fin coincides with the center of the respective circular area of heat input. As in the case of uniform heat production rate in the base plate, the design with 2 fins and uniform fin spacing in the periodic structure has the lowest thermal resistance. A slightly higher thermal resistance is realized by the design 2 fin (b) which shows that equal flow distribution between the parallel fins is more important for the overall thermal performance than an exact placing of the fins above the 
areas of heat input. The topology optimized design has a lower thermal resistance than the best size optimized design. In this case, a relative thermal resistance reduction of $13.6 \%$ is afforded by the topology optimization.

Table 8: Thermal resistance of size optimized parallel fin heat sink designs and the topology optimized design for a pressure drop of $2 \mathrm{~Pa}$ and a non-uniform heat flux in the base plate. Furthermore, the optimal fin thickness is stated for the parallel fin designs.

\begin{tabular}{rrr}
\hline Design & optimal $t h_{\text {fin }}[\mathrm{mm}]$ & $R_{t h}[\mathrm{~K} / \mathrm{W}]$ \\
\hline 1 fin & 0.25 & 267.6 \\
1.5 fin & 0.11 & 197.3 \\
2 fin (a) & 0.07 & $\mathbf{1 8 7 . 0}$ \\
2 fin (b) & 0.07 & 190.1 \\
2.5 fin & 0.05 & 220.6 \\
TO design & - & $\mathbf{1 6 4 . 6}$
\end{tabular}

\subsection{Results model $b$}

As mentioned in section 2.1, the model $b$ geometry is included in this work to generate more complex topologies than those obtained for model $a$ and to demonstrate a case where the number of fins in the optimized designs varies for different pressure drops over the heat sink. Only a uniform heat production rate in the base plate is considered for the model $b$ optimizations. A uniform design field with $\gamma=0.8$ and a straight fluid channel between inlet and outlet are used as initial designs for model $b$ of which the boundary conditions and dimensions are shown in Fig.

2. Hence, fewer initial guesses are used as in the model a optimizations. For this reason, a design continuation approach between the different pressure drops optimized for is applied in the model $b$ optimizations. This means that the optimal design at a certain pressure drop is used as an additional initial design for the next higher and lower pressure drops. As stated in section 5.1 575 a lower solid conductivity in the xy-plane in the thermofluid design layer, i.e. $C_{k}$ value, was chosen in model $b$ compared to model a. This is done to avoid problems with convergence to poor local optima that were observed during preliminary model $b$ optimizations. More specifically, convergence to designs with fewer fins than optimal were observed when using the same $C_{k}$ value as in model $a$. This issue can be avoided when using the presented approach with a lowered $C_{k}$ value and design continuation between different pressure drops; however, the lowered $C_{k}$ value leads to a more academic optimization model. The effect of this assumption can be quantified by comparing the predicted thermal resistance of a pseudo 3D body-fitted mesh validation model with a solid conductivity in the fins corresponding to the conductivity of copper to the thermal resistance of the optimization model as done for model $a$. However, this comparison is omitted for model $b$ as 
this model serves mainly to demonstrate the capabilities of the topology optimization method to generate more complex designs. The unphysically low $C_{k}$ value in the optimization model may possibly also be avoided by other means such as ramping the $C_{k}$ value during the optimization or using different initial designs with several fins as presented for model a but both of these approaches are not considered for model $b$ in this work. An exemplary optimized model $b$ design for $\Delta p=20 P a$ with the corresponding fluid velocity magnitude and streamlines, thermofluid design layer temperature, and base plate temperature is depicted in Fig. 11. The design consists of three fins at the bottom, left, and right boundary of the design domain, where a no slip condition is imposed. Four more fins are formed in the middle of the design domain with small channels between them and one larger channel between the uppermost fin and the symmetry boundary at the top of the design domain. The maximum temperature in the fins is around $51^{\circ} \mathrm{C}$, and it can be seen that the fins are not entirely isothermal in the modeled plane due to the relatively low solid conductivity assumed in model $b$ within the thermofluid design layer. The metal base plate is fairly isothermal with the temperature ranging between $51.9^{\circ} \mathrm{C}$ and almost $52.4^{\circ} \mathrm{C}$.

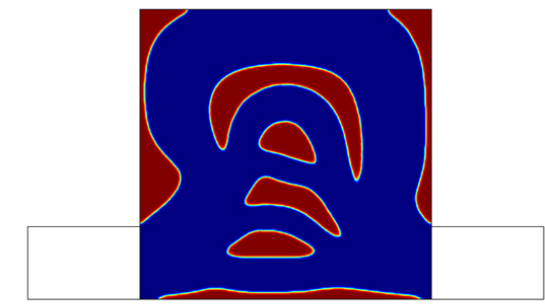

Design

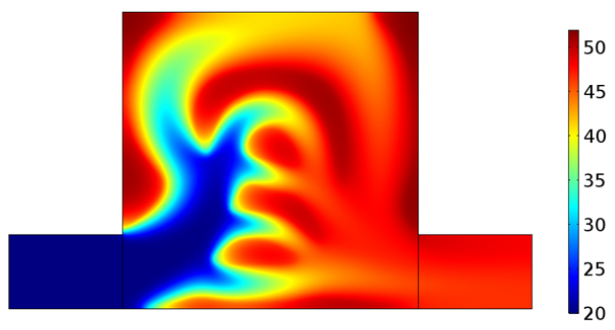

Design layer temperature $\left[{ }^{\circ} \mathrm{C}\right]$

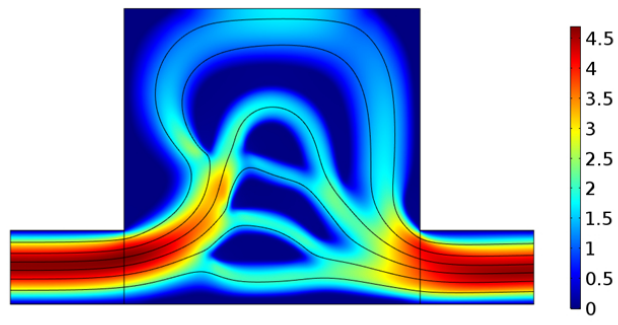

Velocity magnitude $[\mathrm{m} / \mathrm{s}]$ and streamlines

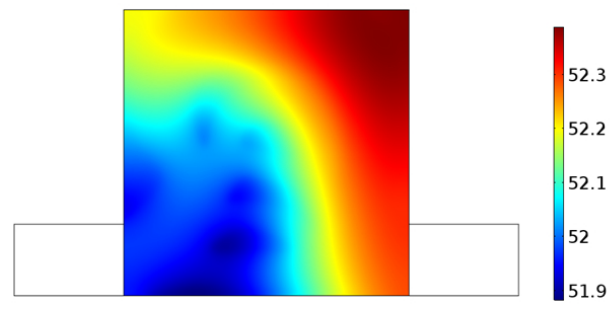

Base plate temperature $\left[{ }^{\circ} \mathrm{C}\right]$

Figure 11: Exemplary model $b$ design obtained for a pressure drop over the heat sink of 20 Pa where red corresponds to solid and blue to fluid. Moreover, the corresponding velocity field, temperature field in the thermofluid design layer, and temperature field in the metal base plate are shown.

Figure 12 shows optimized model $b$ topologies with respect to the pressure drop over the heat sink where pressure drops between 1 and $20 \mathrm{~Pa}$ are considered. The general design is the same in all cases. Three fins are formed at the bottom, left, and right side of the design domain where a no slip condition is imposed. Additionally, a number of fins are formed in the middle of the design domain: One fin for $\Delta p=1 \mathrm{~Pa}$, two fins for $2.5 \mathrm{~Pa}, 3$ fins for $7.5 \mathrm{~Pa}$, and 4 fins for $20 \mathrm{~Pa}$. The 
Reynolds number of the optimized designs defined with regards to the average inlet velocity and inlet width as characteristic length range between 7.2 for $\Delta p=1 \mathrm{~Pa}$ and 67.0 for $\Delta p=20 \mathrm{~Pa}$. Hence, the laminar flow assumption seems to be also well justified for model $b$.

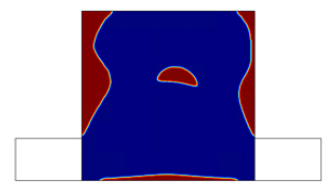

(a)

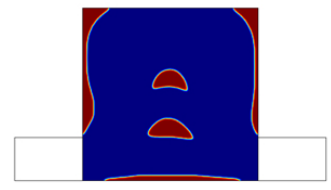

(b)

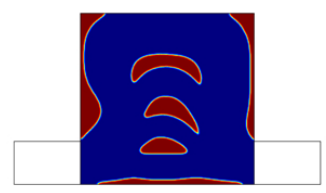

(c)

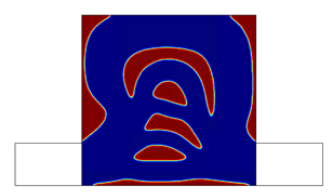

(d)

Figure 12: Influence of pressure drop over model $b$ heat sink on optimized designs. Red corresponds to solid and blue to fluid. The pressure drop takes the values 1 (a), 2.5 (b), 7.5 (c), and $20 \mathrm{~Pa}$ (d).

A cross-check of the objective function values of the optimized designs shown in Fig. 12 based on a pseudo 3D body-fitted mesh validation model is stated in Table 9 The solid-fluid interface is thresholded at $\overline{\tilde{\gamma}}=0.8$ as it is done in model $a$ and the heat sink thermal resistance is computed with two times the heat production rate stated in Table 4 to represent the entire heat sink of which only the bottom half is optimized due to the assumed symmetry boundary condition at the top of the design domain. Moreover, the conductivity of copper is set in the solid domains of the thermofluid design layer to provide a more realistic validation model. Each design performs best at the pressure drop for which it is optimized, which provides some confirmation of the validity of these results from an optimization point of view. It also shows that the presented optimization with lowered $C_{k}$ value may be interpreted as a more heuristic optimization that still yields useful designs when evaluated under more realistic conditions.

Table 9: Discrete cross-check of model $b$ optimized designs heat transfer resistance $[\mathrm{K} / \mathrm{W}]$ based on the pseudo 3D body-fitted mesh validation model.

$\Delta p[\mathrm{~Pa}]$ tested for $\quad \begin{array}{llll}1 & 2.5 & 7.5 \quad 20\end{array}$

\begin{tabular}{rrrrr} 
Design for $\Delta p[\mathrm{~Pa}]$ & & & & \\
\hline 1 & $\mathbf{7 5 1 . 4}$ & 317.8 & 203.1 & 151.4 \\
2.5 & 871.5 & $\mathbf{2 8 8 . 5}$ & 129.6 & 98.2 \\
7.5 & 1216.7 & 360.0 & $\mathbf{1 1 9 . 9}$ & 64.9 \\
20 & 1909.9 & 525.0 & 150.5 & $\mathbf{6 4 . 1}$ \\
\hline
\end{tabular}

\section{Conclusions}

In this work, a thermofluid topology optimization model assuming steady state laminar flow is applied to the design of forced convection air-cooled heat sinks. To reduce the computational effort that is associated with a 3D optimization model, a pseudo 3D heat sink model comprised 
of a 2D modeled heat sink base plate and 2D modeled thermofluid design layer that are thermally coupled is used. Heat sink designs being periodic perpendicular to the flow direction are generated using symmetry conditions at the sides of the modeled domain. The optimization objective is to minimize the heat sink heat transfer resistance for a prescribed pressure drop over the heat sink and prescribed heat production rate in the base plate. Optimized designs are presented and discussed from a thermal engineering point of view. Parametric studies are conducted to analyze the influence of the prescribed pressure drop on the optimized topologies as well as on Reynolds number and thermal resistance of the system. To assess the influence of the implicit solid-fluid boundary representation in density-based topology optimization, 2D and 3D validation simulations with explicit representation of the solid-fluid boundary and a body-fitted mesh are conducted. Moreover, the 3D validation model is used to check the validity of the heat sink representation in the presented pseudo 3D model. A good agreement between the models in terms of predicted thermal resistance and Reynolds number is found over the entire analyzed pressure drop range and reasons for deviations between the models are discussed. It is found that the $3 \mathrm{D}$ thermofluid problem of the airflow around the heat sink fins can be approximated as a $2 \mathrm{D}$ problem in the analyzed case. Still, the pseudo 3D optimization model can also be useful in cases of more pronounced three-dimensional physical phenomena to provide promising starting guesses for a subsequent full 3D optimization. Another means to deal with more pronounced 3D physical phenomena is to add further thermofluid layers to the pseudo 3D model. However, this is nontrivial even if only fin cross-sections being constant in z-direction are considered. This is mainly because assumptions for the heat exchange between the layers, both for fluid-fluid and fin-fin heat transfer, need to be made. Further assumptions would be needed if also momentum transfer between the layers was considered. Moreover, the computational burden increases significantly with each added thermofluid layer. The complexity increases even more if the fin cross-sections are allowed to differ between the layers since a different $2 \mathrm{D}$ design variable field is then needed to be introduced for each thermofluid layer. For these reasons, the analysis of pseudo 3D heat sink models with several thermofluid layers is left for future works. A cross-check based on the 2D validation model shows that each design performs best at the pressure drop optimized for. Furthermore, two exemplary topology optimized designs are benchmarked to a size optimized parallel fin heat sink to provide a comparison to a conventional heat sink geometry. A thermal resistance reduction of up to $13.6 \%$ is found to be afforded by the topology optimization which provides some confirmation for the added benefit of topology optimization. Fins generated with thermal resistance minimization as optimization objective are compared to pressure drop minimized fins for given volume constraints in terms of resulting fin shape, thermal resistance, and pressure drop. Moreover, an exemplary topology optimization is conducted for a model with heat generation in two local thermal hotspots in the base plate. This optimization results in a different topology and 
slightly lower thermal resistance than in the case of a uniform or an almost uniform base plate temperature which demonstrates the usefulness of including the thermal diffusion problem in the

660

base plate in the optimization model. A second non-periodic and more academic, heat sink model with inlet and outlet width smaller than the design domain width is optimized to generate more complex and topologically more interesting fin designs. Optimization results for different pressure drops are presented and discussed and a cross-check based on a 2D body-fitted mesh validation model confirms the optimization technique.

\section{Acknowledgements}

This work was supported by the TOpTEn project sponsored through the Sapere Aude Program of the Danish Council for Independent Research (DFF 4005-00320).

Moreover, the authors wish to thank Rasmus Bjørk for helping to run COMSOL/MATLAB on a computer cluster and Niels Aage and Casper Schousboe Andreasen for their helpful advice with regards to the topology optimization implementation in COMSOL.

\section{References}

[1] S. V. Garimella, A. S. Fleischer, J. Y. Murthy, A. Keshavarzi, R. Prasher, C. Patel, S. H. Bhavnani, R. Venkatasubramanian, R. Mahajan, Y. Joshi, et al., Thermal challenges in nextgeneration electronic systems, Components and Packaging Technologies, IEEE Transactions on 31 (4) (2008) 801-815. doi:10.1109/TCAPT.2008.2001197.

[2] A. Vassighi, M. Sachdev, Thermal and power management of integrated circuits, Springer Science \& Business Media, 2006. doi:10.1007/0-387-29749-9.

[3] A. M. Adham, N. Mohd-Ghazali, R. Ahmad, Thermal and hydrodynamic analysis of microchannel heat sinks: A review, Renewable and Sustainable Energy Reviews 21 (2013) 614622. doi:10.1016/j.rser.2013.01.022.

[4] A. D. Kraus, A. Bar-Cohen, Thermal analysis and control of electronic equipment, Washington, DC, Hemisphere Publishing Corp., 1983, 633 p.

[5] S. Lee, Optimum design and selection of heat sinks, in: Semiconductor Thermal Measurement and Management Symposium, 1995. SEMI-THERM XI., Eleventh Annual IEEE, IEEE, 1995, pp. 48-54. doi:10.1109/STHERM.1995.512051

[6] K. Park, P.-K. Oh, H.-J. Lim, The application of the cfd and kriging method to an optimization of heat sink, International journal of heat and mass transfer 49 (19) (2006) 3439-3447. doi:10.1016/j.ijheatmasstransfer.2006.03.009 
[7] M. P. Bendsoe, O. Sigmund, Topology Optimization: Theory, Methods, and Applications,

[8] J. D. Deaton, R. V. Grandhi, A survey of structural and multidisciplinary continuum topology optimization: post 2000, Structural and Multidisciplinary Optimization 49 (1) (2014) 1-38. doi:10.1007/s00158-013-0956-z.

[9] T. Dbouk, A review about the engineering design of optimal heat transfer systems using topology optimization, Applied Thermal Engineering 112 (2016) 841-854. doi:10.1016/j. applthermaleng.2016.10.134.

[10] O. Sigmund, Design of multiphysics actuators using topology optimization-part i: Onematerial structures, Computer methods in applied mechanics and engineering 190 (49) (2001) 6577-6604. doi:10.1016/s0045-7825(01)00251-1.

[13] A. Iga, S. Nishiwaki, K. Izui, M. Yoshimura, Topology optimization for thermal conductors considering design-dependent effects, including heat conduction and convection, International Journal of Heat and Mass Transfer 52 (11) (2009) 2721-2732. doi:10.1016/j. ijheatmasstransfer.2008.12.013

[14] S.-H. Ahn, S. Cho, Level set-based topological shape optimization of heat conduction problems considering design-dependent convection boundary, Numerical Heat Transfer, Part B: Fundamentals 58 (5) (2010) 304-322. doi:10.1080/10407790.2010.522869.

[15] Y. Joo, I. Lee, S. J. Kim, Topology optimization of heat sinks in natural convection considering the effect of shape-dependent heat transfer coefficient, International Journal of Heat and Mass

[16] E. M. Dede, S. N. Joshi, F. Zhou, Topology optimization, additive layer manufacturing, and experimental testing of an air-cooled heat sink, Journal of Mechanical Design 137 (11) (2015) 111702. doi:10.1115/1.4030989.

[17] M. Zhou, J. Alexandersen, O. Sigmund, C. B. Pedersen, Industrial application of topology 720 optimization for combined conductive and convective heat transfer problems, Structural and Multidisciplinary Optimization 54 (4) (2016) 1045-1060. doi:10.1007/s00158-016-1433-2. 
[18] P. Coffin, K. Maute, Level set topology optimization of cooling and heating devices using a simplified convection model, Structural and multidisciplinary optimization 53 (5) (2016) 985-1003. doi:10.1007/s00158-015-1343-8.

[21] E. M. Dede, Multiphysics topology optimization of heat transfer and fluid flow systems, in: Proceedings of the COMSOL Users Conference, 2009.

[22] G. H. Yoon, Topological design of heat dissipating structure with forced convective heat

[28] X. Qian, E. M. Dede, Topology optimization of a coupled thermal-fluid system under a

19] S. Soprani, J. H. K. Haertel, B. Lazarov, O. Sigmund, K. Engelbrecht, A design approach for integrating thermoelectric devices using topology optimization, Applied Energy 176 (2016) 49-64. doi:10.1016/j.apenergy.2016.05.024.

[20] A. Pizzolato, A. Sharma, K. Maute, A. Sciacovelli, V. Verda, Topology optimization for heat transfer enhancement in latent heat thermal energy storage, International Journal of Heat and Mass Transfer 113 (2017) 875-888. doi:10.1016/j.ijheatmasstransfer.2017.05.098.

transfer, Journal of Mechanical Science and Technology 24 (6) (2010) 1225-1233. doi:10. $1007 / \mathrm{s} 12206-010-0328-1$

[23] E. M. Dede, Optimization and design of a multipass branching microchannel heat sink for 口 electronics cooling, Journal of Electronic Packaging 134 (4) (2012) 041001. doi:10.1115/1. 4007159 .

[24] A. A. Koga, E. C. C. Lopes, H. F. V. Nova, C. R. de Lima, E. C. N. Silva, Development of heat sink device by using topology optimization, International Journal of Heat and Mass Transfer 64 (2013) 759-772. doi:10.1016/j.ijheatmasstransfer.2013.05.007.

[25] T. Matsumori, T. Kondoh, A. Kawamoto, T. Nomura, Topology optimization for fluidthermal interaction problems under constant input power, Structural and Multidisciplinary Optimization 47 (4) (2013) 571-581. doi:10.1007/s00158-013-0887-8.

[26] K. Yaji, T. Yamada, M. Yoshino, T. Matsumoto, K. Izui, S. Nishiwaki, Topology optimization in thermal-fluid flow using the lattice boltzmann method, Journal of Computational Physics 307 (2016) 355-377. doi:10.1016/j.jcp.2015.12.008

[27] K. Yaji, T. Yamada, S. Kubo, K. Izui, S. Nishiwaki, A topology optimization method for a coupled thermal-fluid problem using level set boundary expressions, International Journal of Heat and Mass Transfer 81 (2015) 878-888. doi:10.1016/j.ijheatmasstransfer.2014. 11.005 . tangential thermal gradient constraint, Structural and Multidisciplinary Optimizationdoi: $10.1007 / \mathrm{s} 00158-016-1421-6$ 
[29] C. McConnell, G. Pingen, Multi-layer, pseudo 3d thermal topology optimization of heat sinks, in: Volume 7: Fluids and Heat Transfer, Parts A, B, C, and D, ASME International, 2012. doi:10.1115/imece2012-93093.

[30] J. H. K. Haertel, K. Engelbrecht, B. S. Lazarov, O. Sigmund, Topology optimization of thermal heat sinks, in: Proceedings of the COMSOL Conference 2015, 2015.

[31] J. H. K. Haertel, G. F. Nellis, A fully developed flow thermofluid model for topology optimization of 3d-printed air-cooled heat exchangers, Applied Thermal Engineering 119 (2017) 10-24. doi:10.1016/j.applthermaleng.2017.03.030.

[32] E. Kontoleontos, E. Papoutsis-Kiachagias, A. Zymaris, D. Papadimitriou, K. Giannakoglou, Adjoint-based constrained topology optimization for viscous flows, including heat transfer, Engineering Optimization 45 (8) (2013) 941-961. doi:10.1080/0305215x.2012.717074.

[33] J. Alexandersen, N. Aage, C. S. Andreasen, O. Sigmund, Topology optimisation for natural convection problems, International Journal for Numerical Methods in Fluids 76 (10) (2014) 699-721. doi:10.1002/fld.3954.

[34] J. Alexandersen, O. Sigmund, N. Aage, Topology optimisation of passive coolers for lightemitting diode lamps, in: Proceedings of the 11th World Congress of Structural and Multidisciplinary Optimisation, 2015.

[35] J. Alexandersen, O. Sigmund, N. Aage, Large scale three-dimensional topology optimisation of heat sinks cooled by natural convection, International Journal of Heat and Mass Transfer 100 (2016) 876-891. doi:10.1016/j.ijheatmasstransfer.2016.05.013.

[36] P. Coffin, K. Maute, A level-set method for steady-state and transient natural convection problems, Structural and Multidisciplinary Optimization 53 (5) (2016) 1047-1067. doi: $10.1007 / \mathrm{s} 00158-015-1377-y$

[37] T. Borrvall, J. Petersson, Topology optimization of fluids in stokes flow, International journal for numerical methods in fluids 41 (1) (2003) 77-107. doi:10.1002/fld.426.

[38] M. Stolpe, K. Svanberg, An alternative interpolation scheme for minimum compliance topology optimization, Structural and Multidisciplinary Optimization 22 (2) (2001) 116-124. doi:10.1007/s001580100129.

[39] O. Sigmund, J. Petersson, Numerical instabilities in topology optimization: a survey on procedures dealing with checkerboards, mesh-dependencies and local minima, Structural optimization 16 (1) (1998) 68-75. doi:10.1007/bf01214002 
[40] B. S. Lazarov, O. Sigmund, Filters in topology optimization based on helmholtz-type differential equations, International Journal for Numerical Methods in Engineering 86 (6) (2011) 765-781. doi:10.1002/nme.3072.

[41] F. Wang, B. S. Lazarov, O. Sigmund, On projection methods, convergence and robust formulations in topology optimization, Structural and Multidisciplinary Optimization 43 (6) (2011) 767-784. doi:10.1007/s00158-010-0602-y

[42] Comsol multiphysics 5.2.

[43] O. Schenk, K. Gärtner, Solving unsymmetric sparse systems of linear equations with pardiso, Future Generation Computer Systems 20 (3) (2004) 475-487. doi:10.1016/s0167-739x(03) 00188-2

[44] K. Svanberg, A class of globally convergent optimization methods based on conservative convex separable approximations, SIAM journal on optimization 12 (2) (2002) 555-573. doi: $10.1137 / \mathrm{s} 1052623499362822$

[45] M. Stolpe, K. Svanberg, On the trajectories of penalization methods for topology optimization, Structural and Multidisciplinary Optimization 21 (2) (2001) 128-139. doi: $10.1007 / \mathrm{s} 001580050177$.

[46] E. N. Jacobs, K. E. Ward, R. M. Pinkerton, The characteristics of 78 related airfoil sections from tests in the variable-density wind tunnel, Tech. rep., National Advisory Committee for Aeronautics; Washington, DC, United States (1933).

[47] N. Sahiti, A. Lemouedda, D. Stojkovic, F. Durst, E. Franz, Performance comparison of pin fin in-duct flow arrays with various pin cross-sections, Applied Thermal Engineering 26 (11) (2006) 1176-1192. doi:10.1016/j.applthermaleng.2005.10.042.

[48] F. Zhou, I. Catton, Numerical evaluation of flow and heat transfer in plate-pin fin heat sinks with various pin cross-sections, Numerical Heat Transfer, Part A: Applications 60 (2) (2011) 107-128. doi:10.1080/10407782.2011.588574.

[49] B. S. Lazarov, F. Wang, O. Sigmund, Length scale and manufacturability in density-based topology optimization, Archive of Applied Mechanics 86 (1-2) (2016) 189-218. doi:10.1007/ s00419-015-1106-4.

[50] R. W. Hanks, The laminar-turbulent transition for flow in pipes, concentric annuli, and parallel plates, AIChE Journal 9 (1) (1963) 45-48. doi:10.1002/aic.690090110. 
[51] K. K. Nielsen, K. Engelbrecht, C. R. Bahl, The influence of flow maldistribution on the performance of inhomogeneous parallel plate heat exchangers, International Journal of heat and Mass transfer 60 (2013) 432-439. doi:10.1016/j.ijheatmasstransfer.2013.01.018.

[52] O. Pironneau, On optimum design in fluid mechanics, Journal of Fluid Mechanics 64 (1) (1974) 97-110. doi:10.1017/s0022112074002023

[53] D. W. Kim, M.-u. Kim, Minimum drag shape in two-dimensional viscous flow, International journal for numerical methods in fluids 21 (2) (1995) 93-111. doi:10.1002/fld.1650210202

[54] T. Kondoh, T. Matsumori, A. Kawamoto, Drag minimization and lift maximization in laminar flows via topology optimization employing simple objective function expressions based on body force integration, Structural and Multidisciplinary Optimization 45 (5) (2012) 693-701. doi:10.1007/s00158-011-0730-z. 